\title{
Contribución de la Sociedad Matemática Española a la Educación Matemática en los Veinte Primeros Años de su Fundación
}

\author{
The Contribution of the Spanish Mathematical Society to Mathematics \\ Education during the First Twenty Years to the Foundation
}

Javier Peralta ${ }^{*}$

\begin{abstract}
Resumen
Con ocasión de la reciente celebración del centenario de la fundación de la Sociedad Matemática Española, creada en 1911, se analiza en el artículo si este acontecimiento tuvo repercusiones en la educación matemática en España. Con este objetivo se han consultado la revista instituida con motivo de ese nacimiento: la Revista de la Sociedad Matemática Española, y la Revista Matemática Hispano-Americana, su sucesora. Del estudio de estas publicaciones y de otras fuentes, se concluye que, efectivamente, el nacimiento de la Sociedad Matemática Española dio un impulso a la educación matemática en España, aunque sus efectos habrían de tardar varios años en apreciarse.
\end{abstract}

Palabras-Clave: Sociedad Matemática Española. Centenario. Educación Matemática. Historia de la Educación Matemática Española.

\begin{abstract}
On the recent celebration of the foundation centennial of the Spanish Mathematical Society, created in 1911, we analyze in the paper if this matter had repercussions in Spanish mathematics education. With this goal in mind, we consulted the journal founded because of this birth: the Revista de la Sociedad Matemática Española, and the Revista Matemática Hispano-Americana, its successor. On the study of these publications and other sources, we concluded that, indeed, the birth of Spanish Mathematical Society gave an impetus to mathematics education in Spain, although their effects would not be appreciated until a few years later.
\end{abstract}

Keywords: Spanish Mathematical Society. Centennial. Mathematics Education. History of Spanish Mathematics Education.

\section{Introducción}

A la muerte de Fernando VII (1833), España se halla en un estado cultural lamentable. Sin embargo, a partir de ese momento se abre un periodo esperanzador para la educación y la ciencia en el que se empiezan a crear nuevas instituciones y se elaboran planes

\footnotetext{
* Licenciado y Doctor en Matemáticas, Universidad Complutense de Madrid (UCM). Profesor Titular de Universidad de Matemática Aplicada, Universidad Autónoma de Madrid (UAM), Madrid, España. Dirección postal: Facultad de Formación de Profesorado y Educación, Ciudad Universitaria de Cantoblanco, Calle Francisco Tomás y Valiente, 3, 28049, Madrid, España.E-mail: javier.peralta@uam.es.
} 
de estudio más actualizados (GARMA, 1988). Este movimiento tendrá un mayor empuje en el sexenio democrático (1868-1874), con la influencia posterior de la Institución Libre de Enseñanza (ILE), nacida en 1876, y, muy especialmente, con el ambiente de regeneración nacional surgido a raíz de la crisis del 98.

En el terreno de las matemáticas también se hace patente ese ambiente de progreso (PERALTA, 1999), aunque a finales del siglo XIX nos encontramos con un retraso como de medio siglo con respecto a los países más desarrollados. Nuestros matemáticos más destacados de esa época son José Echegaray y Eizaguirre, insigne ingeniero de Caminos y físico-matemático, ministro de Hacienda y Premio Nobel de Literatura; Eduardo Torroja y Caballé, ilustre geómetra, creador de escuela; Zoel García de Galdeano, que trata de modernizar la matemática española y su enseñanza y funda nuestra primera revista exclusivamente matemática y Ventura Reyes y Prósper, genio polifacético y único matemático español que, en el siglo XIX, publica en una revista matemática extranjera puntera. Los dos primeros desarrollan su magisterio desde la Universidad de Madrid, Galdeano en la Universidad de Zaragoza y Reyes desde el modesto Instituto de Segunda Enseñanza de Toledo. Son los sembradores, los matemáticos de nuestro 98 científico ${ }^{1}$.

Uno de los logros del movimiento antes citado es la creación, en 1900, del Ministerio de Instrucción Pública y Bellas Artes; a lo que hay que añadir la fundación en los años siguientes de dos instituciones capitales para nuestro desarrollo científico: en 1907, la Junta para Ampliación de Estudios e Investigaciones Científicas (JAE) y, en 1908, la Asociación Española para el Progreso de las Ciencias (AEPC). La JAE jugará un importante papel en la formación de investigadores españoles, actuando fundamentalmente en dos frentes: la promoción de estancias en el extranjero (llegó a ser llamada Junta de Pensiones) y la creación de Institutos de investigación. Complementariamente a la JAE, productora de ciencia, la AEPC será el organismo concebido para la difusión de esa ciencia, principalmente mediante la organización de congresos.

En 1911 nace la Sociedad Matemática Española ${ }^{2}$ (SME), que recientemente ha celebrado su centenario. Y con la SME se crea la Revista de la Sociedad Matemática

\footnotetext{
${ }^{1}$ La crisis del 98, producida por la derrota con Estados Unidos y la pérdida de las colonias (Cuba, Puerto Rico...), y la consiguiente reflexión sobre nuestra situación de atraso, suele asociarse únicamente con su importante repercusión literaria: los escritores de la generación del 98 (Azorín, Unamuno, Baroja...). Pero el ambiente de renovación que se produjo también se experimentó en el campo científico; los personajes citados son los matemáticos de ese 98 científico: aquellos que sembraron la semilla en la pobre matemática española de la época, para más tarde recoger su fruto.

${ }^{2}$ En enero de 1929 se le concederá el título de Real, que ha mantenido hasta nuestros días salvo en los años de la República, en los que, lógicamente, renunció a él (GONZÁLEZ REDONDO; LEÓN, 2000).
} 
Española (Rev. SME), que durará de mayo de 1911 a abril de 1917 y a la que sucederá, a partir de 1919, la Revista Matemática Hispano-Americana ${ }^{3}$ (Rev. MHA). Además, en 1915, en el seno de la JAE y a propuesta de la SME, se funda el Laboratorio Seminario Matemático (LSM), nuestro centro de investigación matemático más importante. En esta segunda década de siglo, el relevo de los sembradores lo irán tomando Luis Octavio de Toledo, Cecilio Jiménez Rueda, Miguel Vegas, José María Plans, José Gabriel Álvarez Ude, Esteban Terradas..., y Julio Rey Pastor, auténtico forjador de escuela, que se convertirá en su líder. Ellos recogerán la semilla de la generación anterior y la harán fructificar.

Las anteriores instituciones, y muy especialmente la SME y el LSM jugarán un papel fundamental en el desarrollo matemático español (PINO, 1988), pero aún no ha sido estudiada suficientemente la repercusión de la SME en nuestra educación matemática.

\section{Planteamiento de la investigación. Marco teórico}

El objetivo general del trabajo es analizar, con ocasión de su reciente centenario, si la creación de la SME influyó en nuestra educación matemática; hipótesis que parece razonable, a raíz de lo sucedido con nuestro progreso matemático (PERALTA, 1999).

El marco temporal de nuestro estudio se limitará a los veinte años siguientes a su fundación, que consideramos un lapso de tiempo adecuado para examinar sus posibles repercusiones.

Indagaremos sobre la validez de nuestra hipótesis, esencialmente, analizando las revistas científicas más idóneas: las que se crearon vinculadas a la Sociedad y como consecuencia de su nacimiento, la Rev. SME y la Rev. MHA, que son reflejo de las actividades y evolución de la SME.

Hay que hacer constar, no obstante, que existían, por aquella época, otras revistas científicas que también se ocupaban de matemáticas, aunque no exclusivamente, y que surgieron años antes o años después, llevando una trayectoria independiente de la Sociedad. Básicamente se limitan a estas cuatro: la Revista de la Real Academia de Ciencias Exactas, Físicas y Naturales, continuación de la Revista de los Progresos de las Ciencias Exactas, Físicas y Naturales, nacida en 1851 y que en 1904 cambió su denominación por la primera

\footnotetext{
${ }^{3}$ Tendrá dos series: la primera va del Volumen I (1919) al Volumen VII (1925), y la segunda comienza en el Volumen I (1926) y continúa hasta nuestros días, con el nombre de Revista Matemática Iberoamericana desde 1985.
} 
(como su nombre indica, no trataba solo de matemáticas, sino de ciencias en general, además su proyección didáctica era casi nula); el Boletín de la Institución Libre de Enseñanza (18761936), dirigido a la enseñanza en su conjunto y, en ocasiones, también a la enseñanza de las matemáticas, pero data de treinta y cinco años antes; y las dos siguientes, creadas once años después que la SME: la Revista de las Escuelas Normales (1922-1936), enfocada a la enseñanza primaria en todos sus ámbitos, en la que de vez en cuando escribieron profesores de matemáticas del movimiento normalista (del que más tarde hablaremos) y la Revista de Pedagogía, mayoritariamente de didáctica general (no de didácticas específicas). En vista de todo ello, limitaremos nuestro estudio a la Rev. SME y a la Rev. MHA, como se ha dicho.

Por otro lado, antes de comenzar hemos de hacer dos observaciones. La primera es una aclaración sobre lo que se entendía en ese momento por educación matemática, la segunda se refiere a la significación del nacimiento de la SME en el contexto cultural y científico de la época.

Sobre la primera, hay que precisar que lo que se hacía entonces era estudiar cuestiones aisladas relativas a la enseñanza de las matemáticas, pero sin un planteamiento teórico adecuado. Emplearemos por tanto el término educación matemática en un sentido amplio (como se entendía en las primeras décadas del siglo), pues no es hasta los años setenta (del siglo XX) cuando comienza en España la investigación en lo que hoy se entiende como educación matemática, con su sustrato formal correspondiente. $\mathrm{Y}$ aunque actualmente es un área de conocimiento pujante en España, en el terreno particular de nuestra historia de la educación matemática falta aún mucho por saber. Por ello nos ha parecido interesante investigar, en particular, cómo un hecho tan relevante: la creación de la SME, influyó en nuestra educación matemática (en realidad, según lo dicho, en sus precedentes).

Debe considerarse, asimismo, que la educación matemática surgió en España lentamente de la propia matemática; además, los principales protagonistas en este campo eran precisamente algunos de nuestros mejores matemáticos. ¿Consecuencia?: la existencia de no pocos temas comunes a ambas, sobre los que no parece posible hacer una distinción clara. Estimamos por tanto que no hemos de ceñirnos tan solo al estudio de las cuestiones estrictamente de educación matemática/enseñanza de las matemáticas, sino que también habrá que incluir esos temas comunes. En concreto, la investigación sobre las posibles repercusiones de la creación de la SME en nuestra educación matemática (objetivo general) se abordará desglosando su examen en estos tres aspectos: 
- Cuestiones que podrían contemplarse pertenecientes conjuntamente o limítrofes a matemáticas/educación matemática.

- Publicaciones en educación matemática.

- Instituciones de formación de profesorado y reforma de planes de estudio.

En relación con la segunda observación anunciada más arriba, hay que tener en cuenta que el nacimiento de la SME no fue un hecho aislado, sino que sucedió en un determinado contexto y después de una década en la que se fundaron otras instituciones esenciales para nuestro desarrollo científico y educativo en general. Por ello, para determinar la verdadera dimensión de la influencia de la creación de la SME, parece natural averiguar antes si también dichas corporaciones tuvieron implicaciones en educación matemática. Éste será pues el segundo objetivo de la investigación, aunque, por razones obvias, nos ocuparemos de él en primer lugar. Precisemos en todo caso que se trata de un objetivo secundario, subordinado al más general, explicitado al principio de esta sección.

\section{Fuentes documentales}

Respecto de la heurística de nuestra investigación histórica, esto es, la selección y análisis de fuentes documentales (SALKIND, 1999), digamos que hay abundante literatura sobre los cambios producidos en los planes de estudio en ese periodo, pero muy poca acerca de la evolución de la educación matemática en España en esa etapa. Además, en los trabajos consultados no se vinculan estos hechos con la creación de la SME; por eso, la referencia fundamental para estudiar sus posibles implicaciones ha sido el examen del órgano de difusión de la Sociedad: su revista.

Con más detalle, las fuentes consultadas se catalogan básicamente en los siguientes tipos:

- Libros o artículos escritos por los estudiosos de esos temas:

En una revisión bibliográfica, la información que nos ha parecido más interesante y completa en relación con la situación global de la educación española en esa época ha sido el libro Cien Años de Educación en España reseñado en las referencias, particularmente los capítulos que se ocupan de las enseñanzas secundaria o primaria; junto al de Utande (1964) en la relativa a los planes de estudio. Respecto de nuestro desarrollo matemático en ese periodo, hemos consultado varios artículos de los primeros números de La Gaceta de la Real Sociedad Matemática Española y trabajos 
de Santiago Garma, el autor de estas líneas, etc. Y en cuanto al estudio de la educación matemática, nos ha sido especialmente útil el trabajo de Rico y Sierra (1994), el más completo de los pocos existentes sobre ese tema.

- Con independencia de lo anterior, y casi siempre que nos ha sido posible, hemos acudido a las fuentes originales (y no solo cuando queríamos recabar datos no recogidos por terceros): fundamentalmente la $\operatorname{Rev} . S M E$ y la $\operatorname{Rev}$. MHA, como se ha dicho, e incluso la Gaceta de Madrid (periódico legislativo del Estado, precedente del Boletín Oficial del Estado).

- Por último, para estudiar la proyección pedagógica de la JAE y de la AEPC hemos recurrido principalmente al libro de Martínez (2009) y el artículo de Ausejo (2008), respectivamente; con toda probabilidad los dos trabajos más importantes sobre estos asuntos.

\section{Influencia de las instituciones creadas en la primera década de siglo}

Con la fundación del Ministerio de Instrucción Pública se impulsa una reforma de la educación en todos los niveles, como la creación de las escuelas graduadas, el hito más importante en la renovación de la escuela primaria y una de las banderas del regeneracionismo ${ }^{4}$. Además, se introducen cambios legislativos y reformas de planes de estudio que nos acercan más a Europa.

En el caso de la primera enseñanza, en el inicio del siglo aún estaba vigente prácticamente la Ley Moyano, de 1857, aunque en 1901 se modificará con el Plan Romanones. En la segunda enseñanza, sin embargo, hubo numerosos cambios desde 1857, y en los primeros años después de la creación del Ministerio habrá cuatro nuevas reformas: en 1900, dos en 1901, y en 1903 (UTANDE, 1964). En cuanto a la enseñanza universitaria sucede un hecho muy relevante en el terreno de las matemáticas: el establecimiento de las Ciencias Exactas como licenciatura independiente ${ }^{5}$ (GACETA DE MADRID, 7 de Agosto de 1900); y en 1901 se retocará levemente el plan de 1900 (REVISTA TRIMESTRAL DE MATEMÁTICAS, 1901) que acompañaba a la creación de la licenciatura.

\footnotetext{
${ }^{4}$ No obstante, por falta de fondos, la medida no tiene prácticamente efecto alguno hasta 1911, con la creación de la Dirección General de la Primera Enseñanza y el nombramiento de Rafael Altamira para el cargo, quien relanza el proyecto; a pesar de ello, en 1923 tan solo el 8\% de las escuelas serán graduadas (VIÑAO, 2001).

5 Antes de ello las Facultades de Ciencias constaban de tres secciones: Físico-matemáticas, Físico-químicas y Naturales.
} 
La JAE y la AEPC también participan de una finalidad educativa. Uno de los fines de la JAE, en efecto, es la protección de las instituciones educativas en la enseñanza secundaria y superior (GACETA DE MADRID, 15 de Enero de 1907), y así, respaldará la fundación por el Ministerio de la Escuela Superior de Magisterio (1909) y del Instituto-Escuela de Madrid (1918). En la primera, los maestros recibirán una sólida formación, tanto en materias pedagógicas comunes como en las distintas didácticas específicas, y de ella saldrá un conjunto de normalistas de alta cualificación (en didáctica de la matemática, Margarita Comas, Felipe Saiz, José María Eyaralar...). Del segundo centro, dirigido a la formación del profesorado de segunda enseñanza, hablaremos más adelante.

La AEPC comparte asimismo esa preocupación pedagógica (AUSEJO, 2008), como se deduce al analizar los trabajos presentados en sus distintos congresos. En el primero, celebrado en Zaragoza, en concreto, se abordan diversos problemas referentes a la enseñanza de las matemáticas. Destacan las ponencias: Plan de Enseñanza Matemática y Algunas observaciones pedagógicas acerca de la Matemática, ambas de García de Galdeano, en las que propone una reforma de los planes de estudio, a la vez que realiza interesantes recomendaciones metodológicas y didácticas. Sus consideraciones acerca de la importancia del Análisis matemático, en lo que también inciden los trabajos de Miguel Marzal y Lauro Clariana, influirán en la inclusión, al año siguiente, de una nueva asignatura, Complementos de Cálculo infinitesimal, en el cuarto curso de Ciencias Exactas (GACETA DE MADRID, 14 de Agosto de 1909). Además, en ese congreso Galdeano tratará de canalizar la participación de otros compatriotas en la Commision Internationale de L'Enseignement Mathématique (CIEM), presidida por Poincaré y de la cual Galdeano era el delegado español ${ }^{6}$. En otros congresos de la AEPC, los de Valencia (1910), Granada (1911) y Sevilla (1917), seguirá proponiendo la puesta en marcha de un programa integral de matemáticas que nos aproxime a los modelos internacionales y presentará trabajos de divulgación y sobre aplicación y enseñanza de las matemáticas; en fin, D. Zoel será reconocido internacionalmente con el nombramiento de miembro del Patronato de la revista L'Enseignement Mathématique, junto a Picard, Cartan, Poincaré...

\footnotetext{
${ }^{6}$ Nuestra actividad en el seno de la Comisión fue escasa, y G. Schubring pone a España como ejemplo de uno de los países menos activos o creativos en el campo de la educación matemática (RICO; SIERRA, 1994).
} 


\section{Creación de la Sociedad Matemática Española y sus implicaciones educativas: análisis y resultados}

En el I Congreso de la AEPC antes citado, el general Manuel Benítez Parodi defiende la necesidad de contar con una sociedad de matemáticos y propone su creación, que es aprobada por unanimidad. Se forma, entonces, una comisión promotora formada por Benítez, Octavio de Toledo, Jiménez Rueda y Rey Pastor, que elabora un proyecto, que patrocinará Echegaray y culminará en la creación de la SME, en 1911. La presidencia recaerá en el propio Echegaray, hasta su fallecimiento en 1916, cuando será asumida por García de Galdeano. En cuanto a la Rev. SME, hay una cierta despersonalización en su dirección, pues su edición estará a cargo de un comité de redacción y de una especie de comisión de secretarios de la Sociedad.

Pasemos ya a estudiar las posibles repercusiones de la creación de la SME en nuestra educación matemática/enseñanza de las matemáticas. Hay que decir, en primer lugar, que ése era uno de los cometidos de la Rev. SME, pues en su portada, a continuación del título, se lee: Matemáticas puras y aplicadas. Cuestiones históricas y pedagógicas. Bibliografía y crónica científica. También se desprende del artículo ¡Sursum Corda!, de José Jacobo Durán Loriga, escrito en tono épico en el primer número de la revista - comienza con estas palabras: "La creación de la Sociedad Matemática Española debe señalarse como piedra blanca en los anales de la Ciencia patria" (DURÁN, 1911, p. 21) -, que muestra el entusiasmo con el que es recibida; y en el que, por otra parte, asume que uno de los objetivos de la Sociedad es trabajar en pedagogía matemática, en donde se impone una transformación profunda ${ }^{7}$. Ese entusiasmo, asimismo, se refleja en el número elevado de socios: 423 (algo nada común, incluso hoy), y es también explicable por el hecho de volver a contar con un medio de difusión y en donde poder publicar, puesto que desde 1906 en que deja de editarse la Gaceta de Matemáticas, no existía ninguna revista española exclusivamente matemática (el mayor lapso de tiempo desde la edición de la primera, El Progreso Matemático, en 1891).

Sin embargo, y a pesar de lo dicho anteriormente, esa intencionalidad didáctica no se recoge explícitamente entre los siete fines de la Sociedad. Tan solo el segundo se refiere de algún modo a ello, pero en los siguientes términos: "Estudiar la organización de la enseñanza matemática en los diversos centros oficiales, proponiendo a los Poderes públicos las reformas

\footnotetext{
${ }^{7}$ El artículo ha sido analizado por Peralta (1999).
} 
convenientes, y colaborando en los trabajos de la Comisión internacional de Enseñanza" (REVISTA MATEMÁTICA HISPANO-AMERICANA, 1919, p.197).

Aunque también el cuarto fin muestra un cierto interés educativo: "Organizar concursos varios entre estudiantes de Matemáticas, con premios y estímulos adecuados" (REVISTA MATEMÁTICA HISPANO-AMERICANA, 1919, p.197). O sea, la SME no incluyó explícitamente entre sus objetivos el estudio e investigación en educación matemática (lógico en aquellos años), pero tampoco en la mejora de la enseñanza de las matemáticas (sólo en su organización) o en su metodología y didáctica (pero sí su colaboración con la CIEM), si bien, en su revista tuvieran cabida trabajos en ese campo [concretamente, "cuestiones (...) pedagógicas"].

De acuerdo con la sección 2, analizaremos las implicaciones producidas en cada una de las tres áreas temáticas entonces indicadas, principalmente examinado la Rev. SME y la Rev. MHA. En ambas, cada uno de sus volúmenes consta de diez números (salvo el Volumen VI de la Rev. SME que tiene dos, aunque algo más extensos) y cada número contiene, con ligeras variaciones, las siguientes secciones. En la Rev. SME: Biografías, Sección doctrinal, Notas matemáticas, Artículos diversos, Sección bibliográfica, Crónica, Vocabulario matemático, Intermediario de los matemáticos y Sección de investigación; y en la Rev. MHA, además de la dedicada a artículos, las secciones son: Bibliografía, Cuestiones propuestas, Cuestiones resueltas, Notas y ejercicios elementales, Glosario matemático, Notas necrológicas y Varia ${ }^{8}$.

\subsection{Cuestiones pertenecientes conjuntamente o limítrofes a matemáticas/educación matemática}

Comencemos con la información de libros y revistas (Sección bibliográfica), que es muy extensa; por ejemplo, en el Volumen I de la Rev. SME, n. 1, se recensionan dieciocho libros, la mayoría extranjeros; entre ellos, una Enciclopedia de matemáticas elementales editada por la Sociedad italiana Mathesis. Además, se promueve la traducción al castellano de algunas de las mejores obras extranjeras generalistas y/o divulgativas, idea ya lanzada hacía

\footnotetext{
${ }^{8}$ En 1920 se introduce la sección Consultorio matemático, pero no figura en todos los números; en otros se incluye la sección Retratos; Notas y ejercicios elementales sufre diversos cambios - los Ejercicios elementales se dividen en Ejercicios propuestos y Ejercicios resueltos, y Notas elementales, sólo en algunos números, se denomina Notas didácticas - ; también en algún otro caso aislado se aprecian mínimas variaciones.
} 
tiempo en la Gaceta de Matemáticas Elementales (OCTAVIO DE TOLEDO, 1903; JIMÉNEZ; OCTAVIO DE TOLEDO, 1904). Aunque la propuesta no se hará efectiva hasta la década siguiente a la fundación de la SME, con la publicación de dos colecciones de libros dirigidas por Rey Pastor: "Biblioteca matemática" [en donde se encuentran, Fundamentos de Geometría (1921), de Enriques, Matemática elemental desde un punto de vista superior (1927 y 1931), de Klein...] y "Biblioteca Scientia" [con La teoría de la relatividad al alcance de todos (1925), de Einstein, entre otras obras].

Por otra parte, en la sección Crónica se avisa de la celebración de congresos, premios, fallecimientos etc. Se informa no solo de los Congresos de la AEPC (en España) o de las sesiones de la SME, sino también de eventos en el extranjero, como los Congresos Internacionales de Matemáticos. Por ejemplo, en el Volumen I de la Rev. SME se habla del V Congreso, que se celebrará en Cambridge y, ya finalizado, se vuelve a informar del mismo, así como del número de españoles que han acudido a los cinco primeros, en donde se aprecia claramente su notable incremento en el año posterior al nacimiento de la SME (REVISTA DE LA SOCIEDAD MATEMÁTICA ESPAÑOLA, 1912-1913). Los datos de exponen en el cuadro 1:

\begin{tabular}{|c|c|c|c|c|c|}
\hline Congreso & I. Zurich & II. París & III. Heidelberg & IV. Roma & V. Cambridge \\
\hline Año & 1897 & 1900 & 1904 & 1908 & 1912 \\
\hline Asistentes & 204 & 262 & 336 & 535 & 670 \\
\hline Países & 16 & 27 & 21 & 23 & 27 \\
\hline Españoles & 1 y 1 Señora (!) & 3 & 1 & 5 & 25 y 2 Señoras (!) \\
\hline \multicolumn{7}{|c|}{ Cuadro 1 - Primeros Congresos Internacionales de Matemáticos }
\end{tabular}

También, en el Volumen II, n. 12, Octavio de Toledo, que asiste al V Congreso en representación de Jiménez Rueda (entonces delegado español en la CIEM), comunica cuáles han sido los trabajos de la Subcomisión española, editados conjuntamente en un volumen de 180 páginas. Se presentan en la sección de Enseñanza Matemática y son todos del siguiente estilo: M. Torroja et l'évolution de la Géométrie en Espagne (Miguel Vegas), Les cours d'Analyse mathématique aux Facultés des Sciences espagnoles (Luis Octavio de Toledo), Enseignement des Mathématiques aux Ecoles Normales (Leopoldo Ferreras), etc.

Asimismo, en la sección Intermediario de los matemáticos (inspirada en el boletín francés de ese mismo nombre, fundado en 1894) se constituye un foro de consultas, en espera de respuestas. Para hacerse una idea del tipo de preguntas, se muestran las planteadas en el Volumen I, n. 1 (1911): una se refiere a la construcción de un triángulo dadas sus bisectrices interiores o exteriores, en otra se consulta en qué publicaciones recientes se estudia la continuidad geométrica en relación con las funciones y en la tercera se pregunta si existe 
alguna publicación que trate de la aplicación de la Geometría de la posición a la Dinámica (no todas las consultas, pues, son de matemática superior). Más reveladora es aún la formulada nueve años después por P. Ruiz (REVISTA MATEMÁTICA HISPANO-AMERICANA, v. II, 1920, p. 212): “¿Cuál es exactamente el famoso cuadrado mágico que aparece en uno de sus grabados [de Durero], y qué significado alegórico se le atribuye?"; respuesta a la que responde H. Brocard, y que ya había sido planteada (y contestada) en L'Intermédiaire des Mathématiciens, en 1899.

Otro tema que, creemos, debe incluirse en el bloque que estamos considerando ahora es historia de las matemáticas. Además de la sección Biografías, existen en la revista diversos artículos sobre historia, como los siguientes: La Historia de la Matemática pura en España (REVISTA DE LA SOCIEDAD MATEMÁTICA ESPAÑOLA, v. I, n. 2, 1911-1912) y Unas tablas logarítmicas españolas del siglo XVII (REVISTA DE LA SOCIEDAD MATEMÁTICA ESPAÑOLA, v. IV, n. 36, 1914-1915), de Octavio de Toledo; Tres cuestiones relacionadas con la historia de los logaritmos, de D. Fernández Diéguez (REVISTA DE LA SOCIEDAD MATEMÁTICA ESPAÑOLA, v. VI, n. 52, 1917); ¿Qué debe la matemática a los chinos?, extracto de un artículo de Loria en Bolletino della Mathesis (REVISTA MATEMÁTICA HISPANO-AMERICANA, v. II, n. 6, 1920); etc. Y más concretamente, en relación con la historia de la matemática española, precisamente en el primero de los artículos últimamente citados se propone la elaboración de papeletas bibliográficas descriptivas del mayor número posible de libros de nuestro pasado matemático, y como muestra, Octavio de Toledo presenta algunas en ese mismo trabajo, tarea que más tarde continuarían Vázquez Illá, Torner, Peñalver, Lorente, Artigas y Fernández Diéguez 9 .

Por otra parte, en la sección Vocabulario matemático se desarrolla la idea planteada por Cecilio Jiménez Rueda en el Congreso de la AEPC de Zaragoza (1908) y de la que ya se había hecho eco Octavio de Toledo en el Congreso de la AEPC de Valencia (1910), presentando una primera serie de 369 papeletas como inicio de la elaboración de un Diccionario Matemático.

\subsection{Publicaciones en educación matemática}

En una primera aproximación convendría señalar que la revista incluye en sus páginas numerosos artículos o notas de matemática elemental, esto es, matemáticas de un nivel de

\footnotetext{
${ }^{9}$ En Peralta (2005) puede leerse un comentario sobre la pobre efectividad de la propuesta.
} 
segunda enseñanza o primer curso universitario. Muchos de ellos se reducen a una exposición formal de determinados temas en un contexto matemático predominante y sin una fenomenología didáctica, es más, ni siquiera existen recomendaciones para su enseñanza o reflexiones sobre su aprendizaje; o sea, carecen de consideraciones didácticas oportunas. Sin embargo, en otros se perciben ciertas inquietudes en ese sentido, pues están escritos de modo adecuado para una buena presentación en el aula; creemos incluso que, con una mayor fundamentación teórica, acaso hoy tendrían cabida en la sección a veces denominada Notas de clase en diversas revistas. La lista de unos y otros sería interminable (son más frecuentes aún en los primeros números, en donde escasean artículos de alto nivel matemático), por eso mencionaremos tan solo unos pocos de diversos tomos de la revista.

En el Volumen I (1911-1912) de la Rev. SME: Modo de reconocer si un número es divisible por otro de la forma $a \cdot 10^{n}+1$ ó $a \cdot 10^{n}-1$ (Román Ayza); El interés compuesto (Gabriel Galán); Geometría vectorial. Composición de vectores (E. León y Ortiz)... En el Volumen V (1915-1916): Nuevo procedimiento para multiplicar números enteros (Román Ayza); Método general para ver cuándo un número es divisible por otro en un sistema cualquiera de numeración (Luis Cuevas); Sobre algunas propiedades de los números enteros de dos y tres cifras (J. Jiménez Osuna)... En el Volumen I (1919) de la Rev. MHA: La sucesión de Fibonacci (dividido en cuatro artículos, más otros dos en el Volumen II: Francisco Vera); Volumen de un tetraedro en función de las seis aristas (J. Ruiz-Castizo); Puntos notables de un triángulo (P. M. González Quijano)... En el Volumen V (1923): Sobre la división de un segmento en media y extrema razón (E. Pérez Carranza); Segundo teorema de la media (R. Araujo)... En el Volumen III (1928) de la Rev. MHA, $2^{\mathrm{a}}$ serie: Sobre permutaciones estereométricas. Los cubos policromados (J. Mingot Shelly)... Etc.

En cuanto a artículos sobre enseñanza de las matemáticas en la Rev. SME, solo hemos encontrado alguna pequeña nota en los dos primeros. No es hasta el Volumen III (1913-1914) en donde ya hay cuatro trabajos, incluidos como Artículos diversos sobre Enseñanza Matemática, que son: Congreso de Enseñanza matemática (n. 22), de Octavio de Toledo; Sobre enseñanza matemática (n. 25 y 26), de José Díaz; Congreso de la enseñanza matemática (n.27), de García de Galdeano y Commision internationale de l'enseignement mathématique (n. 28), de Octavio de Toledo. Aunque, como se desprende de sus títulos, tres de ellos son relativos a congresos: el primero es un extracto de los objetivos ${ }^{10}$ y programa del que se va a celebrar en París en abril de 1914, el tercero es el resumen de una conferencia

\footnotetext{
${ }^{10}$ La introducción de las primeras nociones de cálculo diferencial e integral en las escuelas medias -lo que no sucedía en España- y el análisis de la formación matemática de los ingenieros.
} 
dada en la Universidad de Zaragoza por Galdeano, que trata sobre lo acontecido en aquél ${ }^{11}$ y en el cuarto, Octavio de Toledo hace un anuncio de la próxima reunión de la CIEM que se llevará a cabo en Munich en agosto de 1915, invitando a los socios españoles a enviar ideas para que él pueda llevarlas como subdelegado de la Subcomisión española. En fin, la única aportación que no se refiere a un congreso es la segunda, pero no pasa de una reflexión elemental de poco más de una página de Díaz, bachiller y perito (así consta), sobre la enseñanza del Cálculo infinitesimal en las Escuelas Industriales formadoras de los Peritos. En resumen, las cuatro contribuciones tratan de poco más que informaciones de lo que sucede en congresos y reuniones internacionales (en adelante no mencionaremos aportaciones de estos tipos).

En el Volumen V (1915-1916), números 43 y 44, es cuando aparece por primera vez un artículo más específico: se titula Dilatado campo de la investigación matemática, de David Fernández Diéguez, denunciando precisamente la ausencia casi total de trabajos de pedagogía matemática.

Con la Rev. MHA el panorama empieza a cambiar, aunque muy lentamente. Además de las secciones Notas (en las que sí hay cuestiones sobre enseñanza de las matemáticas) y Ejercicios elementales, se encuentran algunos pocos artículos. El primero es: ¿Se puede vulgarizar la matemática superior? (REVISTA MATEMÁTICA HISPANO-AMERICANA, v. I, n. 3, 1919), traducción de otro de R. de Montessus en L'Enseignement Mathématique; pero uno de los más importantes de estos años es el escrito por Galdeano, también en 1919: es un suplemento de la revista, que aparece bajo el epígrafe Sección de crítica, historia, enseñanza y bibliografía matemática. Se trata de un bello y extenso trabajo, primero, sobre lo que podríamos considerar historia y epistemología de la matemática, pero en el que también hay un apartado: El nuevo alcance de la exposición y enseñanza matemática, de doce páginas, en donde aborda distintos problemas de la enseñanza de las matemáticas, y que, sin lugar a dudas, cabría encajar dentro del campo educación matemática/enseñanza de las matemáticas. Luego encontramos Acerca de la enseñanza de la matemática en los institutos de T. Martín Escobar (REVISTA MATEMÁTICA HISPANO-AMERICANA, v. III, n. 6, 1921) y Acerca de la enseñanza de las Matemáticas en las escuelas industriales, del mismo autor (v. VI, n. 5, 1924). En el Volumen VII (1925) existe un avance, al menos desde el punto de vista terminológico, pues desde su número 4 aparece una breve sección denominada Notas

\footnotetext{
${ }^{11}$ Lamenta a este respecto que fuera de España se discuta sobre los contenidos de la segunda enseñanza invadidos por los estudios superiores, mientras que en nuestro país el problema sea, sin embargo, cómo los contenidos de la enseñanza superior son invadidos por los estudios elementales. O sea, pone de manifiesto el bajo nivel de matemáticas en nuestra enseñanza.
} 
didácticas. Entre ellas están: Acerca de la enseñanza de la matemática en los Institutos y la génesis combinatoria de la Aritmética, de J. Mingot (n. 4); Lagunas en la literatura matemática española. Consideraciones acerca de la utilidad y contenido de una Guía para el estudio e investigación de la Ciencia Matemática, de D. Fernández Diéguez (n. 5) y La reforma de la Matemática universitaria en Inglaterra, firmado por las iniciales M. V. (n. 9).

De la segunda serie de la Rev. MHA hemos examinado los cinco primeros tomos (1926 a 1930) y parece que se ha evolucionado favorablemente, aunque ya no figuran Notas didácticas sino Notas elementales. En los Volúmenes I (1926) y V (1930) no vemos nada que reseñar, pero sí en los restantes. En el Volumen II (1927) está el artículo: Algunas consideraciones sobre la formación pedagógica del Profesorado oficial de Matemáticas, de Fernández Diéguez (n. 2), en donde hace una interesante reflexión acerca de la necesidad de una formación psicopedagógica del profesor de matemáticas; en concreto, dice que no es suficiente con saber matemáticas, ser elocuente y exponer con claridad, sino que además hay que enseñarle a enseñar matemáticas, y en esa labor de capacitación docente deben intervenir "la Pedagogía, la Historia y la Filosofía de la Matemática, así como la Psicología Experimental” (FERNÁNDEZ DIÉGUEZ, 1927, p.41). Asimismo en ese número, en la sección Varia aparece el trabajo El nuevo plan de bachillerato, sin mencionar su autor. En el Volumen III (1928) solo hemos descubierto, en Notas elementales, el artículo El número racional, de Manuel Calderón (n. 4). En el Volumen IV (1929) figuran por fin artículos de Pedro Puig Adam: son dos, muy breves, ubicados en la sección de Notas elementales, titulados: Notas sobre pedagogía matemática y Notas sobre la pedagogía de la aritmética elemental.

En el cuadro 2 mostramos las aportaciones mencionadas que consideramos más relevantes en educación matemática publicadas en la Rev. SME y la Rev. MHA:

\begin{tabular}{|c|c|c|c|}
\hline Revista & Volumen & Año & Notas o artículos \\
\hline Rev. SME & $\mathrm{V}$ & $1915-16$ & $\begin{array}{l}\text { Dilatado campo de la investigación matemática (D. Fernández } \\
\text { Diéguez) }\end{array}$ \\
\hline \multirow[t]{5}{*}{ Rev. $M H A$} & I & 1919 & $\begin{array}{l}\text { ¿Se puede vulgarizar la matemática superior? (traducción de un } \\
\text { artículo de L’Enseignement Mathématique de R. de Montessus) }\end{array}$ \\
\hline & I (Suplemento) & 1919 & $\begin{array}{l}\text { El nuevo alcance de la exposición y enseñanza matemática } \\
\text { (apartado de un extenso artículo, Z. García de Galdeano) }\end{array}$ \\
\hline & III & 1921 & $\begin{array}{l}\text { Acerca de la enseñanza de la matemática en los institutos (T. } \\
\text { Martínez Escobar) }\end{array}$ \\
\hline & VI & 1924 & $\begin{array}{l}\text { Acerca de la enseñanza de las matemáticas en las escuelas } \\
\text { industriales (T. Martínez Escobar) }\end{array}$ \\
\hline & VII & 1925 & $\begin{array}{l}\text { Acerca de la enseñanza de la matemática en los Institutos y la } \\
\text { génesis combinatoria de la Aritmética (J. Mingot) } \\
\text { Lagunas en la literatura matemática española. Consideraciones }\end{array}$ \\
\hline
\end{tabular}




\begin{tabular}{|c|c|c|c|}
\hline & & & $\begin{array}{l}\text { acerca de la utilidad y contenido de una Guía para el estudio e } \\
\text { investigación de la Ciencia Matemática (D. Fernández Diéguez) } \\
\text { La reforma de la Matemática universitaria en Inglaterra (M. V.) }\end{array}$ \\
\hline $\begin{array}{c}\text { Rev. MHA } \\
\left(2^{\mathrm{a}} \text { serie }\right)\end{array}$ & $\begin{array}{l}\text { III } \\
\text { IV }\end{array}$ & $\begin{array}{l}1927 \\
1928 \\
1929\end{array}$ & $\begin{array}{l}\text { Algunas consideraciones sobre la formación pedagógica del } \\
\text { Profesorado oficial de Matemáticas (D. Fernández Diéguez) } \\
\text { El nuevo plan de bachillerato (sin autor) } \\
\text { El número racional (M. Calderón) } \\
\text { Notas sobre pedagogía matemática (P. Puig Adam) } \\
\text { Notas sobre la pedagogía de la aritmética elemental (P. Puig } \\
\text { Adam) }\end{array}$ \\
\hline
\end{tabular}

Cuadro 2 - Publicaciones de cierto interés en educación matemática/enseñanza de las matemáticas en la Rev. SME (1911-1917) y la Rev. MHA (1919-1930)

En esta segunda década se va haciendo patente, pues, una mayor tendencia didáctica, en lo que mucho tendrán que ver Rey Pastor y Puig Adam, quienes inician la publicación conjunta de diversos libros escolares de gran calidad, el primero de los cuales es Elementos de Aritmética (1927). A ello hay que añadir los excelentes manuales universitarios de Rey Pastor, que suponen una auténtica renovación en la enseñanza matemática superior: Elementos de Análisis algebraico (1917), Lecciones de Álgebra (1924), Teoría de funciones reales (1925), etc. Y también son hechos reseñables la publicación de la obra Metodología y Didáctica de la Matemática elemental, Tomo I (1933), escrita por Puig Adam y Rey Pastor, y el impulso dado por este último para la creación, en colaboración con la Sociedad Matemática Argentina, de la revista Matemática Elemental (1931-1936), dirigida a estudiantes, en la que tendrá cabida la parte elemental de la Rev. MHA; aunque estos dos últimos hechos ya caen fuera del periodo de tiempo investigado ${ }^{12}$.

\subsection{Formación del profesorado y reforma de planes de estudio}

Del primero de estos asuntos hay que comenzar diciendo que el LSM - íntimamente ligado a la SME - incluía entre sus fines "ser órgano para la transformación de la enseñanza matemática en España, dirigiendo la que se dé en el Instituto-Escuela de Madrid [...], haciendo publicaciones de libros elementales, publicando instrucciones y consejos sobre enseñanza matemática [...]” (LEÓN, 1999, p. 392). Además, la JAE contrataba directamente al profesorado y daba las instrucciones relativas a la organización y objetivos del InstitutoEscuela (MARTÍNEZ, 2009), y en el proceso de selección intervenían organismos dependientes de la JAE, que en el caso de las matemáticas era el LSM. Así pues, el LSM era

\footnotetext{
12 Con anterioridad a los libros mencionados, en el ámbito de la enseñanza primaria y en el contexto del movimiento normalista ya habían aparecido textos didácticos interesantes, como Metodología y Didáctica de la Aritmética de Rodríguez García (1912).
} 
el organismo de la JAE que dirigía académicamente la sección de Matemáticas del InstitutoEscuela, con el que colaboraba directamente (alguno de sus personajes más ilustres, como Álvarez Ude, daría cursos en él).

¿Y qué influencia tuvo la SME en las reformas educativas? Veamos cuál era el panorama. En la Instrucción primaria el Plan Romanones (1901) establecía tres grados (párvulos, elemental y superior), proyectados como ciclos sucesivos en los que se repetían las mismas asignaturas, adaptadas a la edad de los alumnos; esto es, sólo diferenciadas por el carácter cíclico y su adecuación al nivel del curso. El plan duró hasta 1938, y constaba de dos asignaturas de Matemáticas: Aritmética y Nociones de Geometría ${ }^{13}$.

En la segunda enseñanza el Plan Bugallal (1903) no se modificará hasta 1926 (GÓMEZ, 2001). En esta reforma (Plan Callejo) la SME jugará un importante papel en el área de matemáticas, pues la comisión que redactó los programas estaba compuesta por el presidente de la Sociedad (Octavio de Toledo), un vicepresidente (Krahe), un secretario (Plans) y dos vocales (Álvarez Ude y José María Torroja), según consta en el Volumen II (1927), n. 2 de la Rev. MHA, $2^{\text {a }}$ serie . Varían los cursos en los que hay matemáticas: mientras en 1903 se incluía en los cuatro primeros, en el nuevo plan, en el que se hace distinción entre letras y ciencias, están solo en $1^{\circ}, 2^{\circ}$ y $4^{\circ}$, pero para los de ciencias también en $5^{\circ}$ y $6^{\circ}$ (UTANDE, 1964); aunque los contenidos no cambian sustancialmente, pues permanecen los mismos bloques: Aritmética, Geometría, Álgebra y Trigonometría. El plan estará en vigor hasta la llegada de la República.

En cuanto a la sección de Ciencias Exactas de la Universidad, como se ha dicho, existía un plan de estudios de 1900 (retocado en 1901), de cuatro cursos, más uno de doctorado; y en 1909 se incluye, además, la asignatura Complementos de Cálculo infinitesimal en $4^{\circ}$. El plan continúa hasta el curso 1914-1915, en 1915 (GACETA DE

\footnotetext{
${ }^{13}$ El plan Romanones, de 26/10/1901 (Álvaro de Figueroa y Torres, conde de Romanones, era el ministro de Instrucción Pública y Bellas Artes), que dotaría de gran estabilidad a la Instrucción primaria, duplicaba el tiempo de escolaridad obligatoria (de 6 a 12 años, mientras que antes lo era de 6 a 9) y asumía por parte del Estado el pago de las nóminas de los maestros. Con él se pasaba de la escuela unitaria (estando juntos alumnos de distintas edades y capacidades) a la escuela graduada (los alumnos se separaban en clases distintas por edades y niveles), en la que se establecían los tres grados mencionados: párvulos (donde se ubicaban los niños de menor edad, con una enseñanza de carácter más educativo y socializador que instructivo - precedente de lo que hoy suele llamarse educación infantil -), elemental y superior. La enseñanza sería cíclica, variando la intensidad y la extensión de las asignaturas dentro de los grados, así como la distribución y duración de las clases, que serían fijadas en reglamentos posteriores. Las materias que se establecían - de la misma denominación para los grados elemental y superior- eran, además de las dos indicadas de Matemáticas, las siguientes: Doctrina cristiana y Nociones de Historia sagrada; Lengua Castellana; Geografía e Historia; Rudimentos de Derecho; Nociones de Ciencias físicas, químicas y naturales; Nociones de Higiene y de Fisiología humana; Dibujo; Canto; Trabajos manuales y Ejercicios corporales. Como se observa, se trata de un plan enciclopédico y muy ambicioso, dirigido a conseguir una educación integral; sin embargo, quedó más como la expresión de un ideal que de la realidad de lo que sucedería en la inmensa mayoría de las escuelas.
} 
MADRID, 30 de Septiembre) se realizan cambios menores, y en 1917 (GACETA DE MADRID, 9 de Febrero), junto a ligeras variaciones, se establece que en el Doctorado de Ciencias Exactas pueda elegirse la asignatura de Física matemática. En 1920 (GACETA DE MADRID, 1 de Febrero) hay por fin un cambio que parece de importancia en el Doctorado: se incluye la asignatura Metodología y crítica matemática (cuya cátedra será ocupada, por acumulación, por un catedrático de la Facultad propuesto por el Ministerio), que no será obligatoria, sino que podrá elegirse en sustitución de alguna de las otras del Doctorado. Se nombra como catedrático de la misma a Rey Pastor, quien solo aceptó el encargo por un año y gratuitamente, pues la retribución, de 2000 pesetas, fue cedida a la $\mathrm{SME}^{14}$. En el Volumen II, n. 10 de la Rev. MHA (1920), se indica el programa, que consta de nueve temas de investigación, todos históricos ${ }^{15}$.

El 21 de mayo de 1919, Gaceta del 22, se aprueba un Real Decreto sobre autonomía universitaria, que no llega a ponerse en práctica y es derogado tres años después. En un Real Decreto de 9 de julio de 1924, Gaceta del 10, se concede personalidad jurídica a las universidades, y en otro Real Decreto de 19 de Mayo de 1928, Gaceta del 21, se reforman los estudios de las Facultades de Ciencias, que pasan a constar de las secciones de Ciencias Naturales, Ciencias Químicas, Ciencias Físico-Químicas, Ciencias Físicas, Ciencias FísicoMatemáticas y Ciencias Exactas. Ese año, en una Real Orden de 1 de Agosto, Gaceta del 13, y a propuesta de los decanos de Ciencias, se establecen los planes de estudio de dichas Facultades, aunque con la novedad de que cada una de ellas puede añadir alguna asignatura al tronco común fijado por el Ministerio. No diferirán sustancialmente, y lo más destacable de dichos planes, a nuestro juicio, es que en las asignaturas fijadas por el Ministerio no aparece ninguna de Educación matemática, y que en cada uno de los cuatro cursos de licenciatura figura una asignatura de Análisis matemático y otra de Geometría.

\footnotetext{
${ }^{14}$ Las razones de este nombramiento, así como las causas por las cuales Rey Pastor se ocupó de la cátedra solo un año puede encontrarse en Español (2006).

${ }^{15}$ Los temas son los siguientes: 1) Estudio de las extracciones de raíces cuadradas contenidas en la Aritmética de Fr. Juan Ortega, investigando los métodos que hayan servido para obtenerlas; 2) Estudiar las relaciones existentes entre el método de Herón, el de Cataldi y el de Alkasadi con las fracciones continuas ordinarias y la ecuación de Pell; 3) Investigación de los métodos utilizados por Alvaro Thomas en su Liber de triplice motus, París, 1509, para la sumación de series; 4) Reunir indicios suficientes del método descente indefinie de Fermat para reconstruirlo en algunos casos particulares; 5) Análisis de los vestigios del método infinitesimal contenidos en las obras de Pedro Núñez; 6) La introducción del cálculo infinitesimal en España; 7) Estudio sistemático del método de Omerique; 8) Análisis crítico del método de Cauchy para la resolución del problema de Sturm; 9) Análisis de una obra cualquiera representativa de la primera mitad del siglo XIX, con clasificación de sus errores $\mathrm{y}$ faltas de rigor.
} 


\section{Conclusiones}

En resumen, podemos decir que la creación de la SME (y su revista) supuso en el plano educativo los siguientes avances:

- Disponer de un órgano de difusión e información de libros, revistas, reuniones, congresos..., en donde además se plantean preguntas de matemáticas o su enseñanza.

- Favorecer la elaboración progresiva de un diccionario matemático y la información sobre obras matemáticas del pasado.

- La aparición de contribuciones sobre historia de las matemáticas.

- Impulsar la asistencia de matemáticos españoles a congresos internacionales, inicialmente solo con la presentación de trabajos en la sección de Enseñanza matemática.

- Facilitar la publicación de numerosos artículos de matemática elemental (más al principio), o sea, con contenidos matemáticos de nivel de segunda enseñanza o ligeramente superior.

- Incrementar la publicación de trabajos sobre enseñanza de las matemáticas en la Rev. $S M E$, pero que prácticamente no empiezan a surgir hasta 1915. Tienen más componente didáctica y son algo más habituales en la Rev. MHA.

- Propiciar la traducción de obras extranjeras con proyección divulgativa, de autores como Klein o Einstein (aunque no comienzan a publicarse hasta transcurrida una década de la creación de la SME) y, asimismo, la elaboración de manuales escolares y universitarios por Rey Pastor y Puig Adam. Algo después ya se escribe un libro sobre metodología y didáctica de la matemática y se crea la revista Matemática Elemental.

- Participación en la formación del profesorado de enseñanza secundaria mediante una total implicación del LSM (íntimamente ligado a la SME) con el Instituto-Escuela.

- No parece influir en los planes de estudio de primaria, pero sí en los de secundaria (elaboró los planes una comisión compuesta íntegramente por miembros de la junta directiva de la SME). También interviene de algún modo en la planificación de la enseñanza universitaria, dando mayor peso al Cálculo infinitesimal, frente a la preeminencia de las geometrías de Torroja y su escuela (AUSEJO, 2008) en la línea iniciada en 1909, e incorporando la Física matemática en el doctorado de Ciencias Exactas. Es reseñable, además, la inclusión de la asignatura Metodología y crítica 
matemática, en 1920, entre las asignaturas del doctorado, aunque no tratara de metodología de la enseñanza de la matemática, sino de historia.

En suma, el nacimiento de la SME dio un impulso a nuestra educación matemática, aunque la progresión fue lenta y sus efectos tardarían varios años en apreciarse. Ahora bien, de la interpretación de los datos a la luz del análisis realizado, hay que tener en cuenta que, en realidad, la mejora de la enseñanza en general, y de las matemáticas en particular, se inicia con las instituciones creadas en la primera década del siglo (y en la educación primaria incluso antes).

En ese sentido, el nacimiento de la SME significa el final de un proceso y el inicio de otro. El primero tiene su origen en la ILE, la crisis del 98 y el ambiente regeneracionista que surge entonces, y que se concreta en: a) la creación del Ministerio de Instrucción Pública, con sus indiscutibles repercusiones educativas; b) la JAE, organismo fundado para potenciar la investigación y c) la AEPC, y con ella los primeros congresos de matemáticos en nuestro país; las dos últimas, también, con implicaciones sobre la enseñanza. El segundo proceso comienza con el nacimiento de la Sociedad, con el que se abre una nueva senda en la que se dota a la comunidad matemática de una estructura: con un lugar en donde publicar, informar y relacionarse (Rev. SME) y un centro de investigación (LSM); todo ello en el campo de las matemáticas y, en menor grado, en el de la enseñanza de las matemáticas.

La situación puede sintetizarse en la Figura 1:

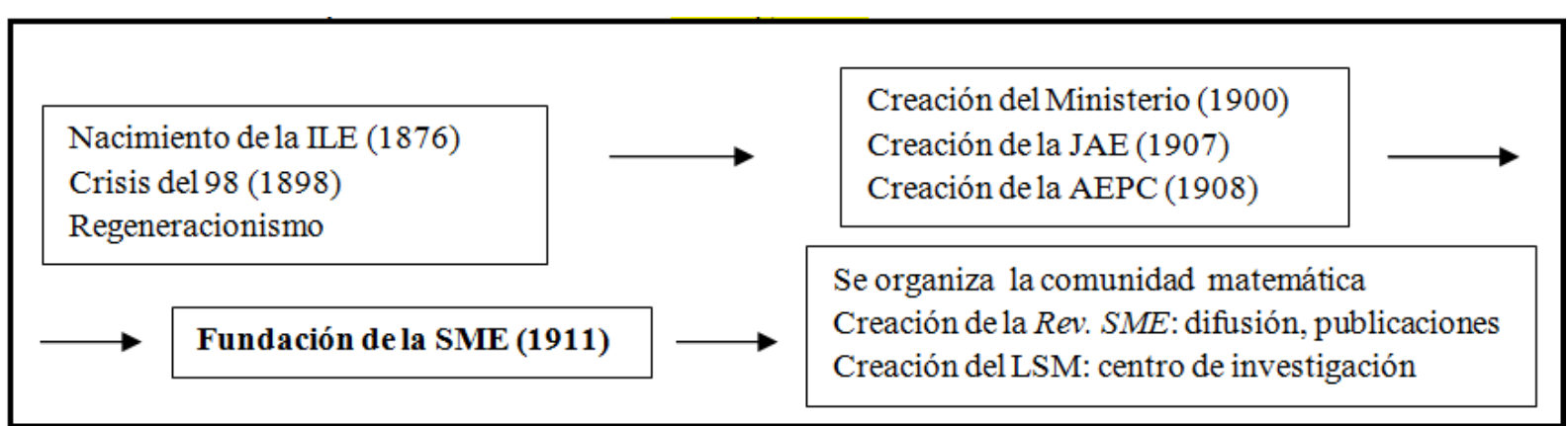

Figura 1 - Fundación de la SME: final de un proceso e inicio de otro

Como consecuencia de estos hechos, el más importante de los cuales sucedido en 1911, la matemática española en la década de los 30 casi se aproximó a la de los países más desarrollados, mientras que la educación matemática comenzó a dar sus primeros pasos. Luego vino la guerra civil y años posteriores, y prácticamente tuvimos que empezar de nuevo (PERALTA, 2006).

\section{Referencias}


AUSEJO, E. La Asociación Española para el Progreso de las Ciencias en el Centenario de su creación. Revista Complutense de Educación, Madrid, v. 19, n. 2, p. 295-310, dic. 2008.

DURÁN, J. J. ¡Sursum Corda! Revista de la Sociedad Matemática Española, Madrid, v. 1, n.1, p. 21-25, may. 1911.

ESPAÑOL, L. Julio Rey Pastor. Primeros años españoles hasta 1920. La Gaceta de la Real Sociedad Matemática Española, Madrid, v. 9, n. 2, p. 564-585, agos. 2006.

GACETA DE MADRID. Madrid, 7 de Agosto de 1900 (Real Decreto del día 3 del Ministerio de Instrucción Pública y Bellas Artes), 15 de Enero de 1907 (Real Decreto del día 11 del Ministerio de Instrucción Pública y Bellas Artes), 14 de Agosto de 1909 (Real Decreto del día 13 del Ministerio de Instrucción Pública y Bellas Artes), 30 de Septiembre de 1915 (Real Decreto del día 25 del Ministerio de Instrucción Pública y Bellas Artes), 9 de Febrero de 1917 (Real Orden de 22 de Enero del

Ministerio de Instrucción Pública y Bellas Artes), 22 de Mayo de 1919 (Real Decreto del día 21 del Ministerio de Instrucción Pública y Bellas Artes), 1 de Febrero de 1920 (Real Orden de 29 de Enero del Ministerio de Instrucción Pública y Bellas Artes) y 21 de Mayo de 1928 (Real Decreto del día 19 del Ministerio de Instrucción Pública y bellas Artes).

GARMA, S. Cultura matemática en la España de los siglos XVIII y XIX. In: SÁNCHEZ RON, J. M. (Ed.). Ciencia y sociedad en España: de la Ilustración a la Guerra Civil. Madrid: CSIC/El Arquero, 1988. p. 93-127.

GÓMEZ, M. N. La enseñanza secundaria pública en España: un antes y un después de la creación del Ministerio de Instrucción Pública y Bellas Artes. In: ÁLVAREZ, P. (Dir.). Cien años de Educación en España. Madrid: Ministerio de Educación, Cultura y Deporte; Secretaría General Técnica, 2001. p. 409-475.

GONZÁLEZ REDONDO, F. A.; LEÓN, M. de. Aproximación a la Historia de las Matemáticas en España. La Real Sociedad Matemática Española. La Gaceta de la Real Sociedad Matemática Española, Madrid, v. 3, n. 2, p. 363-370, agos. 2000.

JIMÉNEZ, C.; OCTAVIO DE TOLEDO, L. Biblioteca Matemática en castellano. La Gaceta de Matemáticas Elementales, Vitoria, v. 2, n. 6, 7 y 8, p. 194-196, ago. 1904.

LEÓN, M. de. Mirando hacia atrás. La Gaceta de la Real Sociedad Matemática Española, Madrid, v. 2, n. 2, p. 389-393, ago. 1999.

MARTÍNEZ, E. Un laboratorio pedagógico de la Junta para Ampliación de Estudios: El Instituto Escuela, sección Retiro de Madrid. Madrid: Biblioteca Nueva, 2009.

OCTAVIO DE TOLEDO, L. Sobre asuntos matemáticos. La Gaceta de Matemáticas Elementales, Vitoria, v. 1, n. 1, p. 27-29, ene. 1903.

PERALTA, J. La matemática española y la crisis de finales del siglo XIX. Madrid: Nivola, 1999.

PERALTA, J. Octavio de Toledo, la sucesión de los promotores de nuestro despertar matemático. La Gaceta de la Real Sociedad Matemática Española, Madrid, v. 8, n. 2, p. 527-547, ago. 2005.

PERALTA, J. Sobre el exilio matemático de la guerra civil española. Hispania Nova, Madrid, v. único, n. 6, p. 582-613, dic. 2006. (Reimpreso en Suma, v. único, n. 56, p. 11-21, nov. 2007 y Suma, v. único, n. 57, p. 9-22, febr. 2008.)

REVISTA DE LA SOCIEDAD MATEMÁTICA ESPAÑOLA. Madrid, v. I, 1911-1912. 
REVISTA DE LA SOCIEDAD MATEMÁTICA ESPAÑOLA. Madrid, v. II, 1912-1913.

REVISTA DE LA SOCIEDAD MATEMÁTICA ESPAÑOLA. Madrid, v. III, 1913-1914. REVISTA DE LA SOCIEDAD MATEMÁTICA ESPAÑOLA. Madrid, v. IV, 1914-1915. REVISTA DE LA SOCIEDAD MATEMÁTICA ESPAÑOLA. Madrid, v. V, 1915-1916. REVISTA DE LA SOCIEDAD MATEMÁTICA ESPAÑOLA. Madrid, v. VI, 1917. REVISTA MATEMÁTICA HISPANO-AMERICANA. Madrid, 1ª serie: v. I y Suplemento, 1919. REVISTA MATEMÁTICA HISPANO-AMERICANA. Madrid, $1^{\text {a }}$ serie: v. II, 1920. REVISTA MATEMÁTICA HISPANO-AMERICANA. Madrid, 1ª serie: v. III, 1921. REVISTA MATEMÁTICA HISPANO-AMERICANA. Madrid, 1ª serie: v. V, 1923. REVISTA MATEMÁTICA HISPANO-AMERICANA. Madrid, 1ª serie: v. VI, 1924. REVISTA MATEMÁTICA HISPANO-AMERICANA. Madrid, 1ª serie: v. VII, 1925. REVISTA MATEMÁTICA HISPANO-AMERICANA. Madrid, 2ª serie, v. II, 1927. REVISTA MATEMÁTICA HISPANO-AMERICANA. Madrid, 2a serie: v. III, 1928. REVISTA MATEMÁTICA HISPANO-AMERICANA. Madrid, 2a serie: v. IV, 1929. REVISTA TRIMESTRAL DE MATEMÁTICAS. Zaragoza, v. I, 1901.

RICO, L.; SIERRA, M. Educación Matemática en la España del siglo XX. In: KILPATRICK, J; RICO, L.; SIERRA, M. Educación Matemática e Investigación, Segunda Parte. Madrid: Síntesis, 1994. p. 99-207.

RODRÍGUEZ GARCÍA, G. Metodología Didáctica de la Aritmética. Madrid: Sucesores de Hernando, 1912.

SALKIND, N. J. Métodos de investigación. México: Prentice Hall, 1999.

UTANDE, M. Planes de estudio de enseñanza media (1787-1963). Madrid: Ministerio de Educación Nacional, Dirección General de Enseñanza Media, 1964.

VIÑAO, A. La escuela graduada: una nueva organización escolar y pedagógica. In: ÁLVAREZ, P. (Dir.). Cien Años de Educación en España. Madrid: Ministerio de Educación, Cultura y Deporte; Secretaría General Técnica, 2001. p. 363-388. 